\title{
Rare decays at LHCb
}

\section{Jaroslaw Wiechczynski*i}

Institute of Nuclear Physics, Polish Academy of Science, Cracow (Poland)

E-mail: jaroslaw.wiechczynskidifj.edu.pl

LHCb has a wide ranging programme of searches for New Physics in rare decays of $B$ and $D$ mesons. Recent highlights are presented, including results of the angular analysis of the decay $B^{0} \rightarrow K^{* 0} \mu^{+} \mu^{-}$and photon polarization in the $B^{+} \rightarrow K^{+} \pi^{-} \pi^{+} \gamma$ process. We also report about the observation of a resonance in the decay $B^{+} \rightarrow K^{+} \mu^{+} \mu^{-}$at low recoil.

XXII. International Workshop on Deep-Inelastic Scattering and Related Subjects,

28 April - 2 May 2014

Warsaw, Poland

* Speaker.

†n behalf of the LHCb collaboration. 


\section{Introduction}

Experimental searches for a New Physics (NP) may be performed in direct and indirect way, i.e. by observing the decay products of new heavy particles produced in high energy collisions or by making precise measurements of decay processes that can be sensitive to the presence of new virtual particles. In the latter case, the important field are rare $B$ decays involving $b \rightarrow s$ transitions. They are Flavour Changing Neutral Curents (FCNC) processes and are forbidden in the Standard Model (SM) at tree level, they proceed via penguin and box diagrams. New possible virtual particles may occur in the diagrams loops and modify several observables like differential branching fractions, angular variables, $\mathrm{CP}$ asymmetries etc. Such processes are useful to probe high energy scale (much higher than for direct searches).

The LHCb experiment [1] is a dedicated tool for studying b-hadron decays. The large $b \bar{b}$ and $c \bar{c}$ cross sections at the LHC supported by the unique acceptance range, efficient trigger and excellent vertexing and particle identification of the LHCb detector, makes a perfect enviroment for studying of a large spectra of different final states. It is then very well suited to study rare decays.

In this article we briefly present the results of $B^{0} \rightarrow K^{* 0} \mu^{+} \mu^{-}$decay studies [2][3], $c \bar{c}$ spectroscopy analysis of $B^{+} \rightarrow K^{+} \mu^{+} \mu^{-}$at low recoil [4] and the first observation of the photon polarization in the $B^{+} \rightarrow K^{+} \pi^{-} \pi^{+} \gamma$ decay [5].

\section{Measurement of form-factor independent observables and angular analysis of the decay $B^{0} \rightarrow K^{* 0} \mu^{+} \mu^{-}$}

The rare decay $B^{0} \rightarrow K^{* 0} \mu^{+} \mu^{-}$is a FCNC b $\rightarrow \mathrm{s} l^{+} l^{-}$transition, where $B \rightarrow K^{* 0}$ amplitudes depend on Wilson Coefficients and accounts for short distance effects (sensitive for New Physics). In extensions of the SM, contributions from new particles can enter in competing amplitudes and modify the differential branching fraction and angular distributions of the decay products. In particular, the $B^{0} \rightarrow K^{* 0} \mu^{+} \mu^{-}$decay is sensitive to the Wilson coefficients $C_{9}$ and $C_{10}$ assigned to the vector and axial-vector semileptonic operators. On the other hand, form-factors related to the long distance effects are significant source of theoretical uncertainty, hence it is crucial to construct the
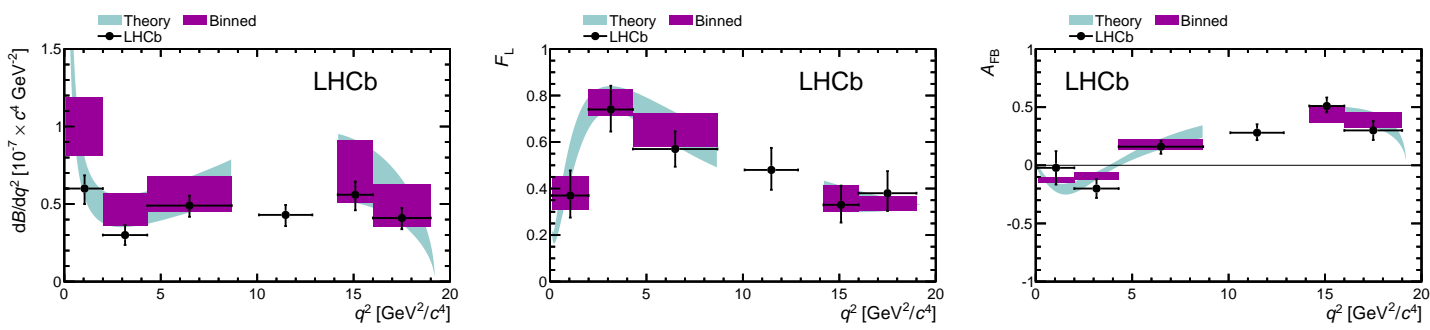

Figure 1: Differential branching fraction (left), fraction of longitudinal polarisation of the $K^{* 0}, F_{\mathrm{L}}$ (center) and dimuon system forward-backward asymmetry, $A_{\mathrm{FB}}$ (right) of the $B^{0} \rightarrow K^{* 0} \mu^{+} \mu^{-}$decay as a function of the dimuon invariant mass squared. The data are overlaid with a SM prediction for the decay (light-blue band) [6]. A rate average of the SM prediction across each $q^{2}$ bin is indicated by the dark (purple) rectangular regions. No SM prediction is included in the region close to the narrow $c \bar{c}$ resonances. 
special observables that minimize these effects. The presented analyses are performed on $1 \mathrm{fb}^{-1}$ sample of data and described in detail in Refs. [2,3].

Differential branching fraction and the angular analyses are performed in six intervals of $q^{2}$ (invariant mass squared of the dimuon system). The number of signal candidates in each of the $q^{2}$ bins is estimated by performing an extended unbinned maximum likelihood fit to the $K^{+} \pi^{-} \mu^{+} \mu^{-}$invariant mass distribution. Differential branching fraction is obtained by normalizing $K^{* 0} \mu^{-} \mu^{+}$yield to the reference $B^{0} \rightarrow J / \psi K^{* 0}$ yield and it is compared to the theoretical predictions (Fig.1(left)). The results are compatibile with SM predictions.

The angular distribution is described by two helicity angles $\left(\theta_{\ell}, \theta_{K}\right)$, the angle $\phi^{1}$ between the decay planes of the $K^{* 0}$ and the dimuon system in the $B^{0}$ meson rest frame, and dimuon invariant mass squared, $q^{2}$ :

$$
\begin{aligned}
\frac{1}{\mathrm{~d} \Gamma / \mathrm{d} q^{2}} \frac{\mathrm{d}^{4} \Gamma}{\mathrm{d} q^{2} \mathrm{~d} \cos \theta_{\ell} \mathrm{d} \cos \theta_{K} \mathrm{~d} \hat{\phi}}=\frac{9}{16 \pi}[ & F_{\mathrm{L}} \cos ^{2} \theta_{K}+\frac{3}{4}\left(1-F_{\mathrm{L}}\right)\left(1-\cos ^{2} \theta_{K}\right)- \\
& F_{\mathrm{L}} \cos ^{2} \theta_{K}\left(2 \cos ^{2} \theta_{\ell}-1\right)+ \\
& \frac{1}{4}\left(1-F_{\mathrm{L}}\right)\left(1-\cos ^{2} \theta_{K}\right)\left(2 \cos ^{2} \theta_{\ell}-1\right)+ \\
& S_{3}\left(1-\cos ^{2} \theta_{K}\right)\left(1-\cos ^{2} \theta_{\ell}\right) \cos 2 \hat{\phi}+ \\
& \frac{4}{3} A_{\mathrm{FB}}\left(1-\cos ^{2} \theta_{K}\right) \cos \theta_{\ell}+ \\
& \left.A_{9}\left(1-\cos ^{2} \theta_{K}\right)\left(1-\cos ^{2} \theta_{\ell}\right) \sin 2 \hat{\phi}\right] .
\end{aligned}
$$

The parameters $F_{\mathrm{L}}$ (fraction of the longitudinal polarization of $K^{* 0}$ ), $A_{\mathrm{FB}}$ (forward-backward asymmetry of the dimuon system), $S_{3}$ and $A_{9}$, as functions of $K^{* 0}$ amplitudes, depend on the Wilson Coeficients and thus can probe NP in the studied decays. These parameters are extracted by the simultaneous fit to $\left(\cos \theta_{\ell}, \cos \theta_{K}, \phi\right)$ and invariant $B$ mass distributions. The fitted $F_{\mathrm{L}}$ and $A_{\mathrm{FB}}$ parameters in six $q^{2}$ bins are presented in Fig.1(center and right) and the results are consistent with the SM expectations.

The forward-backward asymmetry changes sign in the SM at a well defined value of $q^{2}, q_{0}^{2}$, whose position is free from form-factor uncertainties. Experimentally we obtain $q_{0}^{2}=4.9 \pm 0.9$ $\mathrm{GeV}^{2} / c^{4}$, which is perfectly consistent with $\mathrm{SM}\left(q_{0(S M)}^{2}=4.36_{-31}^{+33} \mathrm{GeV}^{2} / c^{4}\right.$ [7]).

In addition, the modified angular obsevables were proposed [8] with reduced form-factor uncertainties by using certain transformations applied to the specific regions of the three-dimensional
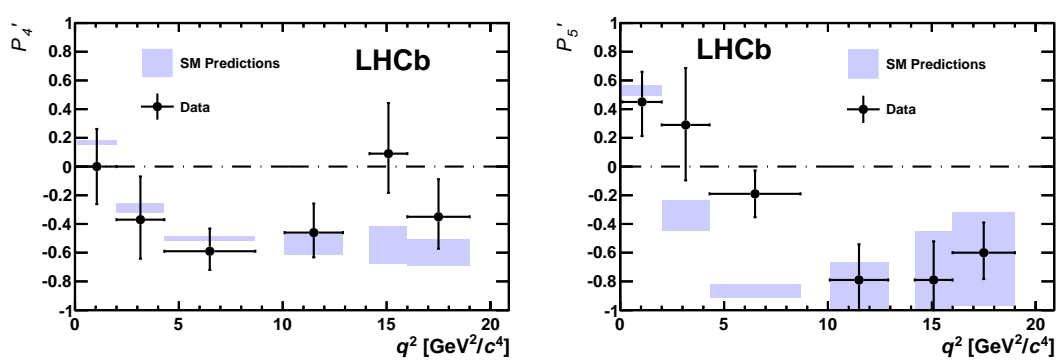

Figure 2: Measured values of $P_{4}^{\prime}$ and $P_{5}^{\prime}$ (black points) compared with SM predictions [9] (blue bands).

\footnotetext{
${ }^{1}$ the following formula assumes the transformation: $\hat{\phi}=\phi+\pi$ for $\phi<0$
} 
angular space. These transformations exploit the (anti)-symmetries of the differential decay rate with respect to combinations of angular variables.

Measured values of two exemplary variables $\left(P_{4}^{\prime}, P_{5}^{\prime}\right)$ are presented in Fig.2 compared to the theoretical expectations. We observe local deviation from the SM for the interval $\left(4.30<q^{2}<8.68\right)$ $\mathrm{GeV} / c^{2}$ that might be interpreted by the NP affecting Wilson Coefficient $C_{9}$. Possible explanations also include the presence of the heavy flavour changing $Z^{\prime}$ boson [10].

\section{Observation of a resonance in the $B^{+} \rightarrow K^{+} \mu^{+} \mu^{-}$decay}

We present dedicated analysis of $B^{+} \rightarrow K^{+} \mu^{+} \mu^{-}$at low recoil (high $q^{2}$ region), above the open-charm threshold. Motivation of this study is the observation of higher charmonium resonances, which apear in the tree level diagrams and compete with the nonresonant FCNC loops. It is essential to understand these broad and overlaping states when interpreting NP contribution to the FCNC decays. This analysis is based on the data sample of $3 \mathrm{fb}^{-1}$ [4].

The invariant mass of the dimuon system $m\left(\mu^{+} \mu^{-}\right)$for the decay $B^{+} \rightarrow K^{+} \mu^{+} \mu^{-}$is shown in Fig. 3. Its resolution is improved by the kinematic fit with $K^{+} \mu^{+} \mu^{-}$mass constrained to the nominal $B$ mass. We consider resonant component and non-resonant $B^{+} \rightarrow K^{+} \mu^{+} \mu^{-}$signal, while the later one contains both axial-vector and vector contribution. The additional component describes the interference between the resonant part and the vector component of non-resonant $B \rightarrow K^{+} \mu \mu$ contribution. The influence of constructive interference is crucial for the observation of the resonances in the dimuon mass system. Three charmonium resonances are taken into account: $\Psi(3770)$ (at the low edge), $\Psi(4040)$ and $\Psi(4160)$ (which was previously observed by BES experiment [11]).

The mean and width values of the $\Psi(4160)$ resonance are measured to be $4191_{-8}^{+9} \mathrm{MeV} / \mathrm{c}^{2}$ and $65_{-16}^{+22} \mathrm{MeV} / c^{2}$, respectively, where the uncertainties include statistical and systematic contributions. We provide the first observation of the decay $B^{+} \rightarrow \Psi(4160) K^{+}, \Psi(4160) \rightarrow \mu^{+} \mu^{-}$ and (with the assumption of the lepton universality) the respective branching fraction: $B F(B \rightarrow$ $\left.\Psi(4160) K^{+}\right)=\left(5.1_{-1.2}^{+1.3} \pm 3.0\right) \times 10^{-4}$. We find the signal of $\Psi(4040)$ to be not significant, while the contribution from the $\Psi(4160)$ state is around $20 \%$ of the total signal in the high $q^{2}$ region.

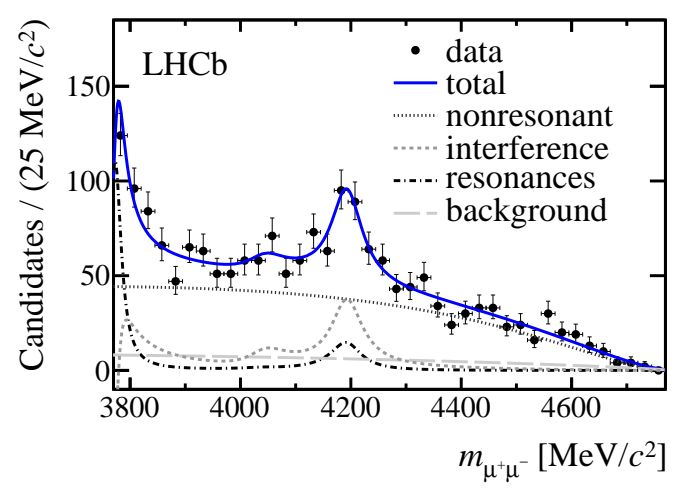

Figure 3: Dimuon mass distribution with fit results overlaid. Presented contributions include the nonresonant vector and axial vector components, interference terms and the $\psi(3770), \psi(4040)$ and $\psi(4160)$ resonances. 


\section{Radiative $B^{+} \rightarrow K^{+} \pi^{-} \pi^{+} \gamma$ decay}

Radiative $B^{+} \rightarrow K^{+} \pi^{-} \pi^{+} \gamma$ decay proceeds via $b \rightarrow s \gamma$ transition including the electroweak penguin loop. The photon is predominantly left-handed in the SM, however, in several extensions of SM the significant right-handed contribution is expected, for instance if a heavy fermion contributes in penguin loop. So far, there was no direct observation of the non-zero photon polarization in such decays.

In this analysis based on $3 \mathrm{fb}^{-1}$ of data [5], we measure the angle $(\theta)$ of the photon with respect to the plane of three hadrons $\left(K^{+} \pi^{-} \pi^{+}\right)$in their centre-of-mass frame, in different intervals of $K^{+} \pi^{-} \pi^{+}$mass. From this measurement we evaluate the up-down asymmetry between number of events on each side of this plane.

The differential decay rate, as a function of the hadron momenta and the $\theta$ angle might be expressed as:

$$
\frac{\mathrm{d} \Gamma}{\mathrm{d} s \mathrm{~d} s_{13} \mathrm{~d} s_{23} \mathrm{~d} \cos \theta} \propto \sum_{i=0,2,4} a_{i}\left(s, s_{13}, s_{23}\right) \cos ^{i} \theta+\lambda_{\gamma} \sum_{j=1,3} a_{j}\left(s, s_{13}, s_{23}\right) \cos ^{j} \theta,
$$

where $s_{i j}$ and $s$ are functions of the four-momenta of the $K^{+} \pi^{-} \pi^{+}$hadrons, $a_{j}$ are functions depended on the resonanses (and their interference) present in the given range of $K^{+} \pi^{-} \pi^{+}$mass and $\lambda_{\gamma}$ is a polarization of the photon. The up-down asymmetry of the photon direction with respect to the $K^{+} \pi^{-} \pi^{+}$plane is given by $\mathscr{A}_{u d} \equiv\left(\int_{0}^{1} \mathrm{~d} \cos \theta \frac{\mathrm{d} \Gamma}{\mathrm{d} \cos \theta}-\int_{-1}^{0} \mathrm{~d} \cos \theta \frac{\mathrm{d} \Gamma}{\mathrm{d} \cos \theta}\right) /\left(\int_{-1}^{1} \mathrm{~d} \cos \theta \frac{\mathrm{d} \Gamma}{\mathrm{d} \cos \theta}\right)$ and depends only on odd powers of $\cos \theta$, thus is proportional to the photon polarisation.

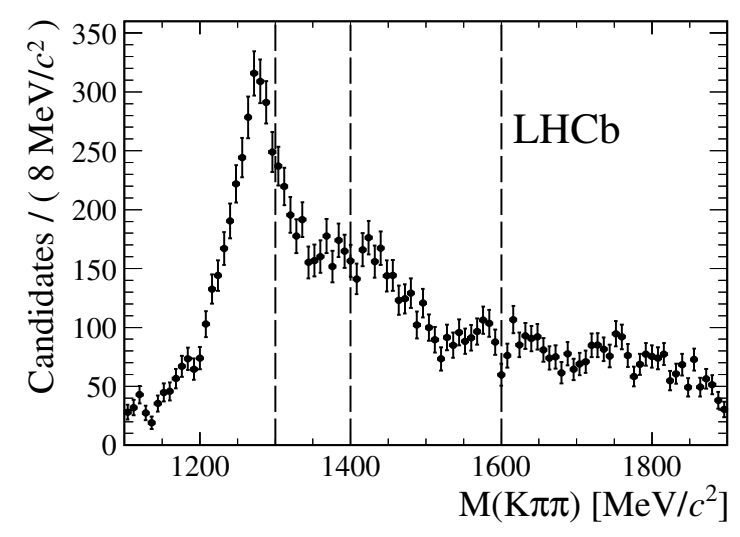

Figure 4: Background-subtracted $K^{+} \pi^{-} \pi^{+}$mass distribution of the $B^{+} \rightarrow K^{+} \pi^{-} \pi^{+} \gamma$ signal. Vertical dashed lines separates the four intervals of interest.

By performing the maximum likelihood fit to the $B$ mass candidate we extract background subtracted $K^{+} \pi^{-} \pi^{+}$invariant mass distribution (Fig. 4). This spectra includes interference of several resonances (ex. $K_{1}(1270), K_{1}(1400), K^{*}(1410), K_{2}^{*}(1430), K_{2}(1580), K_{2}(1770)$ ), which cannot be easily separated. This gives the theoretical limitations for extracting the value of $\lambda_{\gamma}$ from the asymmetry measurement. We perform inclusive measurement in four intervals of $K^{+} \pi^{-} \pi^{+}$ mass, as indicated in Fig. 4.

Fitting the $B$ mass candidate in separate bins of $\theta$ angle we obtain background subtracted angular distributions for each bin of the $K^{+} \pi^{-} \pi^{+}$mass (Fig.5). Those distributions are fitted with 

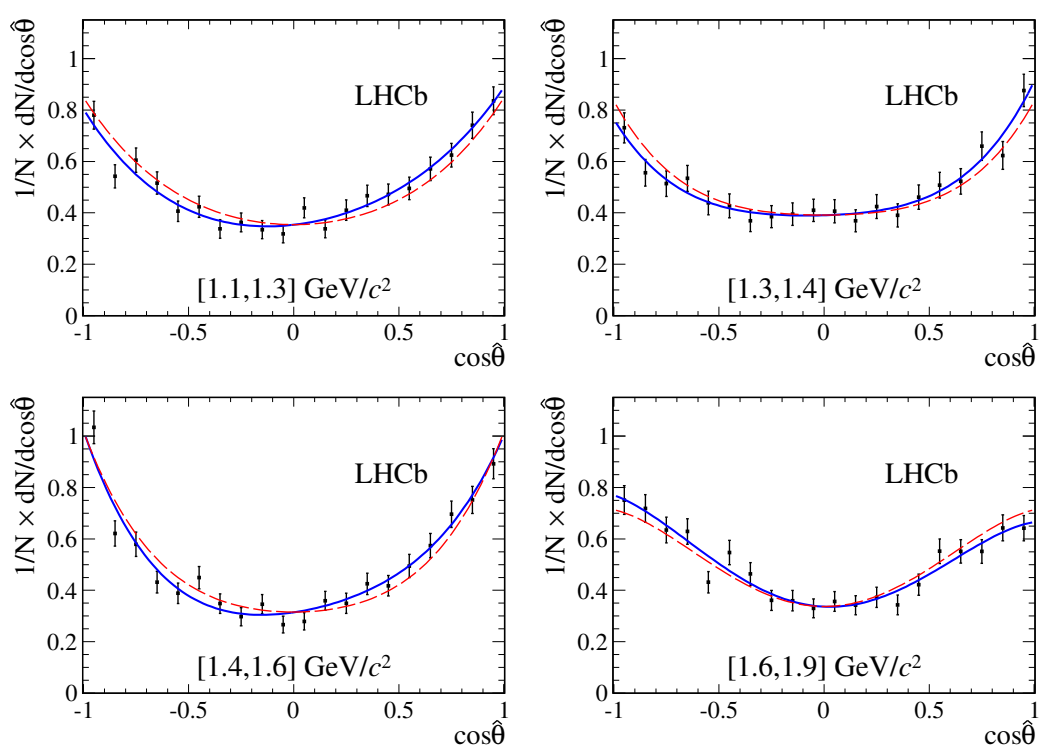

Figure 5: Distributions of $\cos \theta$ for $B^{+} \rightarrow K^{+} \pi^{-} \pi^{+} \gamma$ signal in four intervals of $K^{+} \pi^{-} \pi^{+}$mass. The solid blue (dashed red) curves are the result of fits allowing all (only even) Legendre components up to the fourth power.

the formula $\mathrm{f}\left(\cos \theta ; c_{i}\right)=\sum_{i=0}^{4} c_{i} L_{i}(\cos \theta)$, where $L_{i}$ are Legendre polynomials. The coefficients $c_{i}$ are determined and given to aid with theory developments. The up-down asymmetry is extracted from the odd $c_{i}$ 's and is found to be different from zero at the significance level of 5.2 $\sigma$. This is the first observation of a parity-violating photon polarization in the radiative $B$ decays, however, better understanding of the $K^{+} \pi^{-} \pi^{+}$mass structure is crucial to interpret the measurement in terms of the photon polarisation.

\section{References}

[1] LHCb Collaboration, The LHCb Detector at the LHC, 2008 JINST 3 S08005.

[2] LHCb collaboration, JHEP 08 (2013) 131, arXiv:1304.6325.

[3] LHCb collaboration, Phys. Rev. Lett. 111 (2013) 191801, arXiv:1308.1707.

[4] LHCb collaboration, Phys. Rev. Lett. 111 (2013) 112003, arXiv:1307.7595.

[5] LHCb collaboration, Phys. Rev. Lett. 112, 161801 (2014), arXiv:1402.6852.

[6] http://arxiv.org/abs/1105.0376.

[7] M. Beneke, T. Feldmann, and D. Seidel, Eur. Phys. J. C41 (2005) 173, arXiv:hep-ph/0412400.

[8] S.Descotes-Genon et al. JHEP, 1305:137 (2013), arXiv:1303.5794.

[9] LHCb Collaboration, JHEP 05 (2013) 137, arXiv:1303.5794.

[10] arXiv:1307.5683; arXiv:1308.1501; arXiv:1310.1082; arXiv:1311.6729.

[11] BES Collaboration, Phys. Lett. B 660, 315 (2008). 